\title{
Toi, moi... et les autres ? Musique populaire et migration dans le film musical Toi, moi, les autres d'Audrey Estrougo (2011)
}

\author{
Renaud LAGABRIELLE (Vienne)
}

\section{Summary}

In my article, I analyse the relationship between famous French songs and migration in the French film musical Toi, moi, les autres (Audrey Estrougo 2011), which mixes the codes of the fairy tale romcom with a story about undocumented migrants in contemporary Paris. I underscore that the use of these songs - very few of the musical arrangements are sung by figures of sans-papiers - is pernicious. They serve to highlight and to cement the love story of the two protagonists much more than to develop a real political discourse. I argue that the filmmaker has exploited the memorial - and sentimental - dimension of the French songs for the benefit of the romantic story but has failed to offer a critical discourse on the situation of the undocumented migrants, which had been one of the intentions of the film.

Deuxième long métrage ${ }^{1}$ de la réalisatrice et scénariste française Audrey Estrougo, Toi, moi, les autres, sorti sur les écrans français en 2011, s'inscrit dans le corpus encore assez maigre et méconnu de films que je propose de désigner par l'appellation générique ( films en chanté ?, à savoir des films dans lesquels alternent texte parlé et texte chanté, ce dernier faisant partie intégrante du récit filmique (cf. Lagabrielle 2016). Dans cette version moderne et contemporaine de l'histoire du prince et de la bergère, Audrey Estrougo voulait lier le côté romantique du conte de fées et la critique sociale et politique, critique qui concerne ici la question de la migration, en particulier celle des sans-papiers. La réalisatrice parle elle-même à propos de son film d'un « conte social " (Dossier de presse 2011, 3). L'histoire de Toi, moi, les autres est de fait caractéristique des comédies romantiques ou comédies contes de fées, s'inscrivant par-là dans une tendance du cinéma français depuis le milieu des années 1990 (cf. Harrod 2015, 1-13) : reprenant les codes génériques de la comédie musicale conte de fées hollywoodienne (cf. Altman 1992), le film relate l'histoire d'amour d'une jeune fille immigrée d'un milieu populaire, Leilla, interprétée par Leïla Bekhti, et d'un jeune homme de la haute 
bourgeoisie parisienne, Gabriel, joué par Benjamin Siksou. Cette histoire, qui n'est pas sans rappeler celle de West Side Story (Robert Wise et Jerome Robbins, 1961), est entremêlée à une seconde trame narrative, la situation de sans-papiers africains à Paris - le personnage central des sans-papiers, Tina (interprétée par Marie-Sohna Condé), est une Sénégalaise vivant et travaillant en France depuis des années avec sa fille Sally (Cheïna Correa Lafaure).

Le film reprend des chansons préexistantes, des chansons populaires du répertoire français des années 1950 à 2000, de "Quand on n'a que l'amour » de Jacques Brel (1956) à «La bonne étoile " de Mathieu Chédid (2003), jouant ainsi avec la dimension patrimoniale et mémorielle de la chanson française ${ }^{2}$, avec ce que Phil Powrie propose d'appeler des «sons de mémoires » (Powrie 2014, 59). Ces chansons, qui ont été réorchestrées pour le film, sont toutes interprétées par les acteurs et les actrices. Mais parmi ces chansons, dix au total, seules cinq sont associées plus ou moins explicitement, dans la narration du film, à la question de la migration et des sans-papiers :

"Tout le monde" (Zazie 2003)

«Un autre monde » (Téléphone 1984)

«Sauver l'amour» (Daniel Balavoine 1985)

"Quand on n'a que l'amour» (Jacques Brel 1956)

"Avec nos armes ", qui a été composée pour le film par Gladys Gambie (2010) et qui constitue la musique du générique de fin.

Et parmi ces cinq chansons, deux seulement concernent uniquement la trame narrative de la situation des sans-papiers, "Tout le monde » et " Un autre monde ». Cette dernière est d'ailleurs la seule chanson dont les canteuses (Hirschi 2008, 20) soient des sans-papiers. Comme nous le verrons en effet, les paroles et/ou la mise en scène des trois autres chansons renvoient certes à la question des sans-papiers, mais elles sont également étroitement associées à l'histoire d'amour de Leïla et Gabriel, voire effacées par elle.

Cet effacement, pour ne pas dire écrasement, se trouve d'ailleurs dans la composition même de l'affiche du film, qui sera reprise pour la pochette du DVD, affiche sur laquelle l'image rend explicite ce que le titre du film ne faisait que suggérer : au premier plan, un gros plan de Leïla et Gabriel, enlacés, et, en petit à l'arrière-plan, d'autres personnages à peine identifiables - une composition qui renvoie au < toi > et au < moi > définis d'une part, aux ( autres `, une masse indéfinie de gens, d'autre part.

Je me propose donc dans ce qui suit d'étudier les relations entre chanson (populaire, aux deux sens du terme) et migration dans Toi, moi, les autres, en m'attachant notamment à démontrer que ce nombre faible de chansons associées à la narration des sans-papiers est révélateur de l'équilibre précaire qui lie cette dernière à celle de l'histoire d'amour des deux jeunes personnages. Nous verrons que malgré la dimension politique que peuvent revêtir certaines des chansons, la volonté de la réalisatrice d'aborder des questions liées aux sanspapiers dans un film qui est avant tout une comédie romantique, représente un exercice 


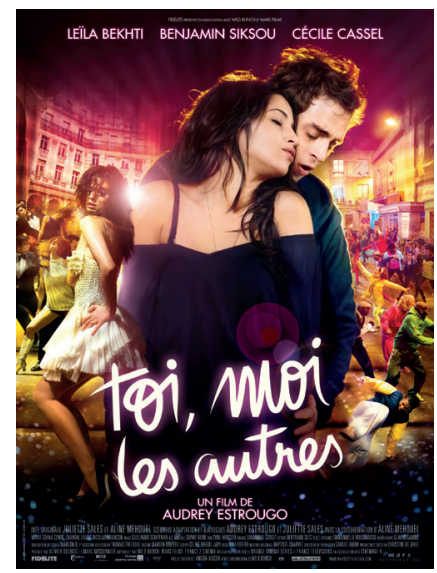

Figure 1. Affiche du film

d'équilibre sur la corde raide, exercice dont - c'est la thèse de cet article - Estrougo n'a pas su surmonter les défis.

Comme je l'ai rappelé en introduction, la construction narrative de Toi, moi, les autres repose sur les codes et les conventions du genre de la comédie musicale conte de fées hollywoodienne, notamment de l'âge classique, tels que les a analysés Rick Altman dans son ouvrage pionnier La comédie musicale hollywoodienne. Les problèmes de genre au cinéma (1992). Parmi ces conventions génériques reprises dans le film d'Estrougo, la plus importante est sans doute la structure biplex (dual-focus narrative) de la narration. Toi, moi, les autres repose en effet sur une dualité à plusieurs niveaux. La narration repose sur " un couple de stars de sexe opposé et se réclamant de valeurs divergentes " (Altman 1992, 30), et " une opposition secondaire accompagne cette partition sexuelle principale ", les " caractéristiques secondaires étant, au départ, toujours diamétralement antithétiques et réciproquement exclusives " (Altman 1992, 36) : Leïla et Gabriel appartiennent on l'a vu à deux milieux sociaux différents et alors qu'elle incarne le labeur et l'engagement, Gabriel, lui, représente l'oisiveté et l'égoïsme. Ces oppositions sont explicitement signifiées dès le générique de début du film, construit en montage alterné et avec de nombreux écrans partagés qui font ressortir les contrastes existant entre les deux jeunes personnages et leurs milieux respectifs.

Selon les codes et conventions du genre, la trame narrative première, l'histoire d'amour, est entremêlée à une seconde trame narrative qui met par ailleurs en danger cette histoire d'amour. Dans Toi, moi, les autres, les obstacles à l'histoire d'amour de Leilla et Gabriel ont une dimension particulière. En effet, le père de Gabriel (Nicolas Briançon) est le préfet de police de Paris, et en tant que tel, c'est lui qui décide de l'expulsion de Tina et de sa fille. Et Gabriel doit promettre à son père d'épouser Alexandra (Cécile Cassel), qu'il avait quittée pour Leïla, s'il veut que son père fasse en sorte que Tina et sa fille ne soient pas expulsées - ce que le père d'ailleurs ne fera pas. 
L'histoire du film se passe dans la France contemporaine, à l'époque où Nicolas Sarkozy était président de la République, et le film n’hésite pas à déployer une critique plus ou moins explicite de la politique migratoire française de ces années-là : on voit une image de Nicolas Sarkozy à la Préfecture de police ; le père de Gabriel, un homme désagréable, porte le prénom de Brice, comme le ministre de l'Intérieur puis ministre de l'Immigration, de l'Intégration et de l'Identité nationale de l'époque, Brice Hortefeux, connu notamment pour être inflexible dans la question de la régularisation des sans-papiers ; le personnage du film prononce d'ailleurs des mots similaires à ceux qu'avait prononcés Hortefeux à propos des Arabes : "Quand il y en a un, ça va, c'est quand il y en a beaucoup qu'il y a des problèmes. " (Cf. Johannès 2010) De même, dans un cauchemar, Gabriel se voit marié à Alexandra qui appelle leurs enfants Nicolas, Carla et Cécilia. Enfin, l'église Saint Bernard, dans laquelle s'étaient réfugiés quelques centaines de sans-papiers en 1996 avant d'en être expulsés, est montrée plusieurs fois dans le film, dans des (échelles de) plans qui ne laissent aucun doute sur l'importance attribuée à ce lieu devenu symbolique.

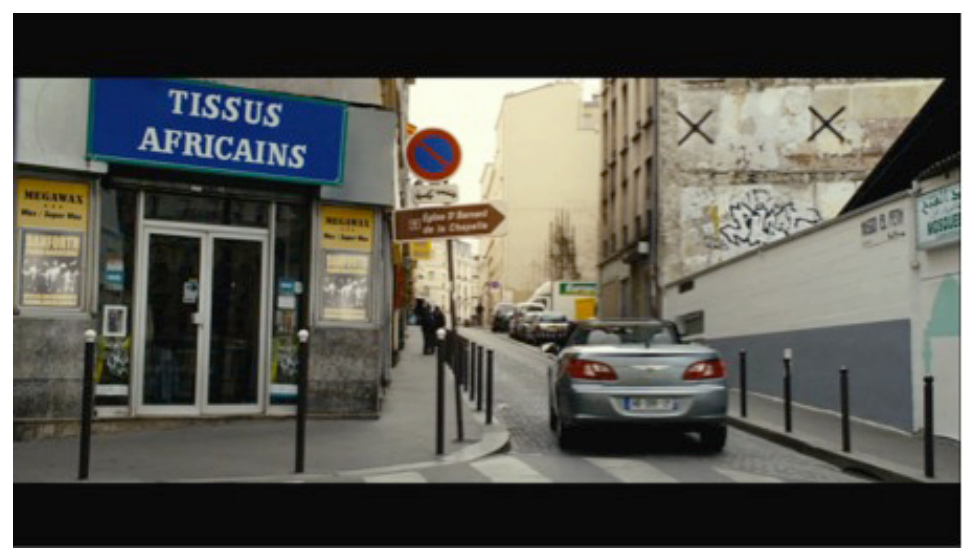

Figure 2. Pancarte indiquant "Église Saint Bernard de la Chapelle » (2:52)

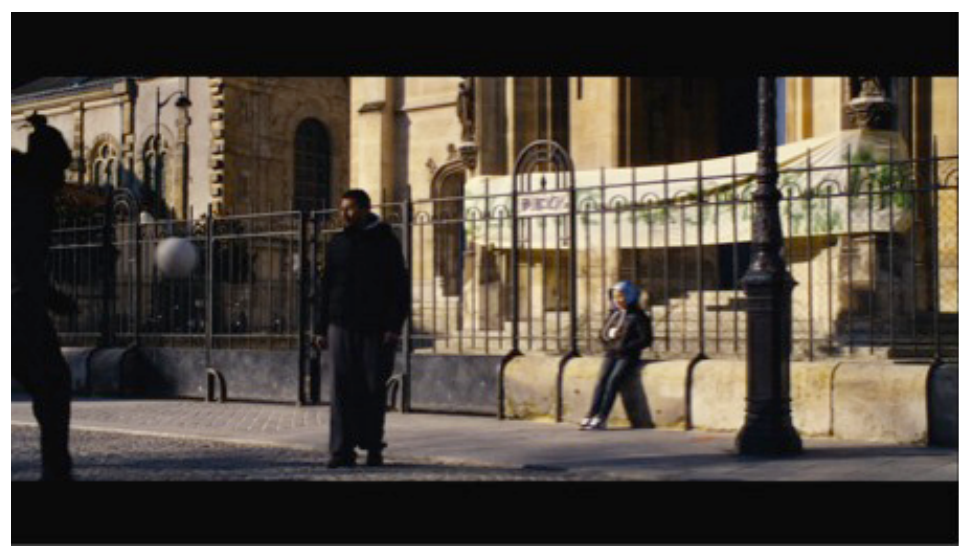

Figure 3. L'église Saint Bernard de la Chapelle en plan de demi-ensemble (1:00:13) 
Enfin, dans la dernière séquence du film, la réalisatrice a également intégré des images d'archives de cette expulsion ainsi que de manifestations de sans-papiers.

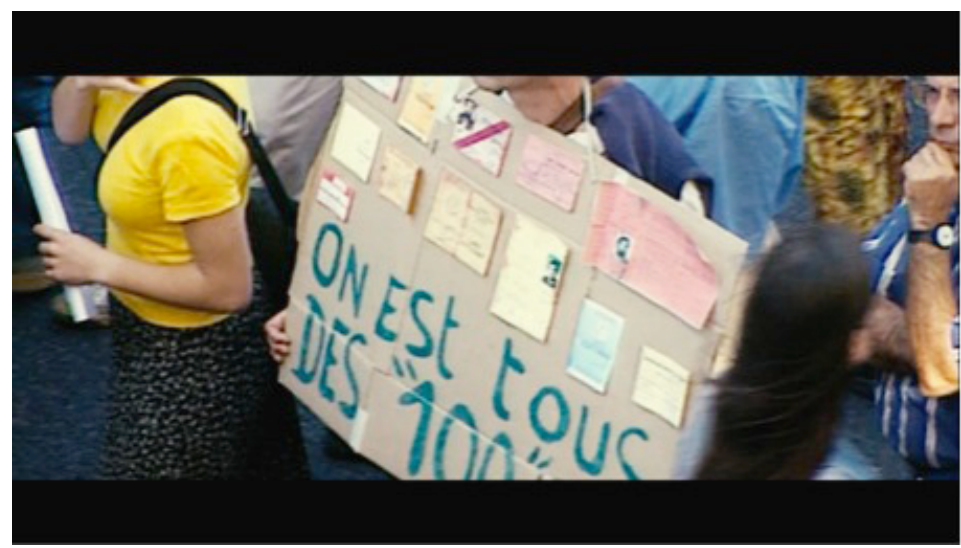

Figure 4. Images d'archives d'une manifestation pour les droits des sans-papiers (1:19:25)

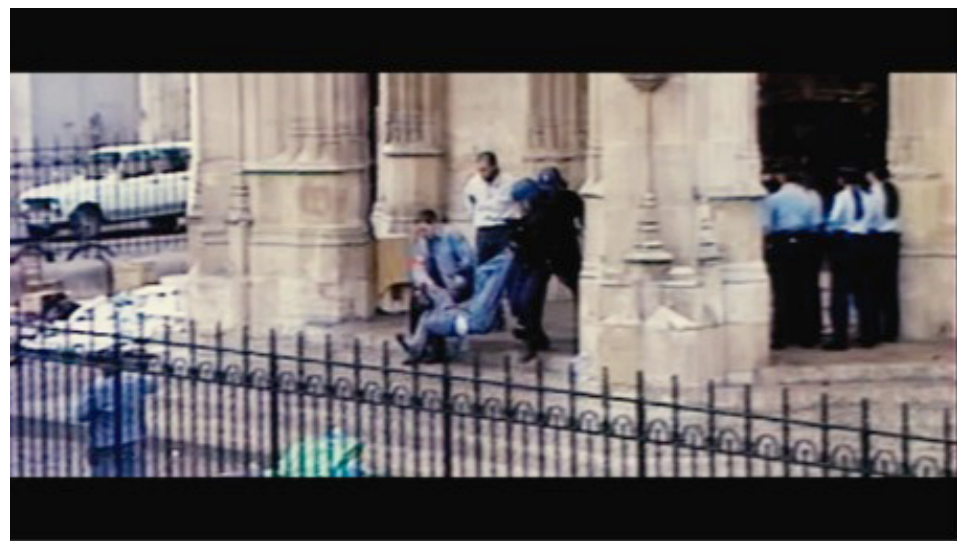

Figure 5. Image d'archives de l'expulsion des sans-papiers (1:19:52)

Malgré cette dimension critique ainsi que la volonté évidente du film de dénoncer les injustices faites aux sans-papiers vivant en France, la réalisation montre la difficulté qu' il y a à traiter ces injustices. Ceci apparaît dès la deuxième scène chantée, dans laquelle les personnages interprètent une chanson de Jacques Dutronc de 1962, «Le temps de l'amour " (interprétée à l'époque par Françoise Hardy). Leîla est seule avec son amie Tina dans le salon de coiffure dans lequel celle-ci travaille, et on apprend par leur dialogue que Tina et sa fille Sally sont en situation irrégulière en France, et que Leïla s'occupe de leur dossier de régularisation. Alors que les deux femmes, au début de la scène, parlent de ce dossier, la discussion dévie sur la rencontre récente de Leïla et Gabriel. Au fur et à mesure de la scène, l'importance de l'histoire d'amour en germe apparait beaucoup plus grande que celle du dossier de Tina et de sa fille, la question des sans-papiers étant complètement reléguée au second plan puis 
finalement totalement effacée, comme le suggère le fait que Tina commence d'entonner "Le temps de l'amour ", l'interprétation de la chanson occupant ensuite l'intégralité de la scène. Soulignons ici le mode d'insertion de la chanson, qui est le même pour l'ensemble des chansons du film : il s'agit, selon la terminologie proposée par Jérôme Rossi, du «mode scénique ", la chanson fait partie de l'univers diégétique des personnages et les paroles sont intelligibles aussi bien pour ces derniers que pour les spectateurs (Rossi 2010, 69).

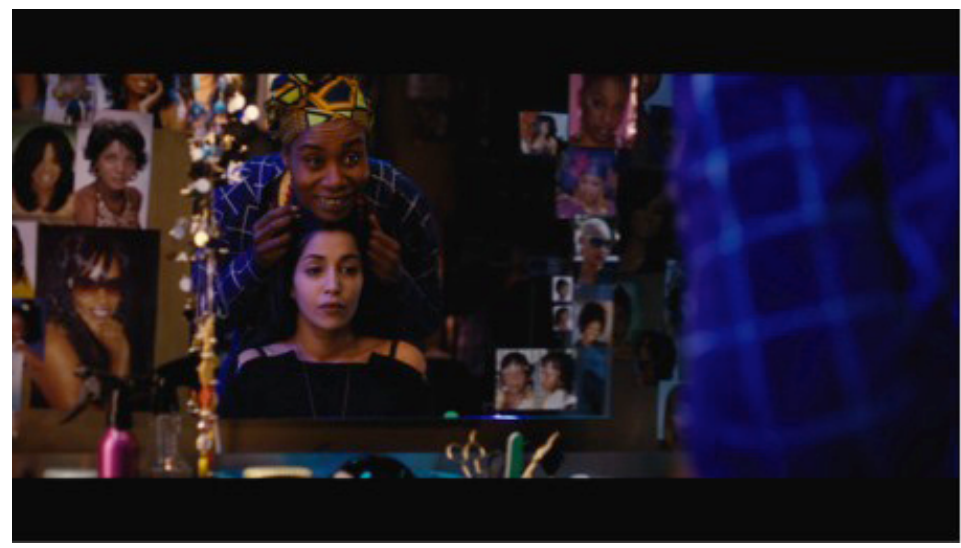

Figure 6. Tina et Leïla interprétant « Le temps de l'amour » (15:17)

Comme je l'ai précisé plus haut, seules deux chansons sont, dans la narration du film, associées à la migration en général, aux sans-papiers en particulier. La première, qui fait suite à celle discutée à l'instant, est "Tout le monde ", de Zazie, composée en 2003. Le choix d'une chanson de Zazie n'est bien sûr pas anodin, puisque cette chanteuse est connue pour son engagement social - notamment au sein de Sol en Si et des Enfoirés pour les Restos du Cœur -, et "Tout le monde " s'adresse directement à Jean-Marie Le Pen (Fontana 2007, 204). Il s'agit là de la première chanson dans laquelle chantent de nombreux personnages issus de la migration, permettant ainsi à une "voix plurielle ", phénomène narratif caractéristique des communautés marginalisées (cf. Wagner 2006), de se déployer. Et la chute de la chanson, sur une structure en chiasme, est bien porteuse du message politique que la chanson veut faire passer : "Tout le monde il est grand / Assez grand pour tout l'monde. " Mais cette chanson reflète pour moi la manière dont est abordée la question des sans-papiers dans son ensemble, à savoir de manière un peu naïve. ${ }^{3}$ "Tout le monde il est beau, tout le monde il est gentil »... Cette s simplicité > mine quelque peu l'effet du discours qui se veut critique, et se retrouve d'ailleurs dans des déclarations pathétiques des deux personnages principaux. Leïla rétorque par exemple à une employée de la préfecture qu'elle ne lui parle pas de "numéros mais d'êtres humains ", faisant écho à Gabriel qui explique à son père que "ce ne sont pas des dossiers, mais des hommes ".

Notons ici qu'alors que dans le cas des deux premières chansons, l'intégration du texte chanté dans la narration se faisait sur un mode naturaliste, sans chorégraphie de danse, 
"Tout le monde » relève quant à lui du numéro musical tel qu'on le connaît avant tout des comédies musicales hollywoodiennes et bollywoodiennes.

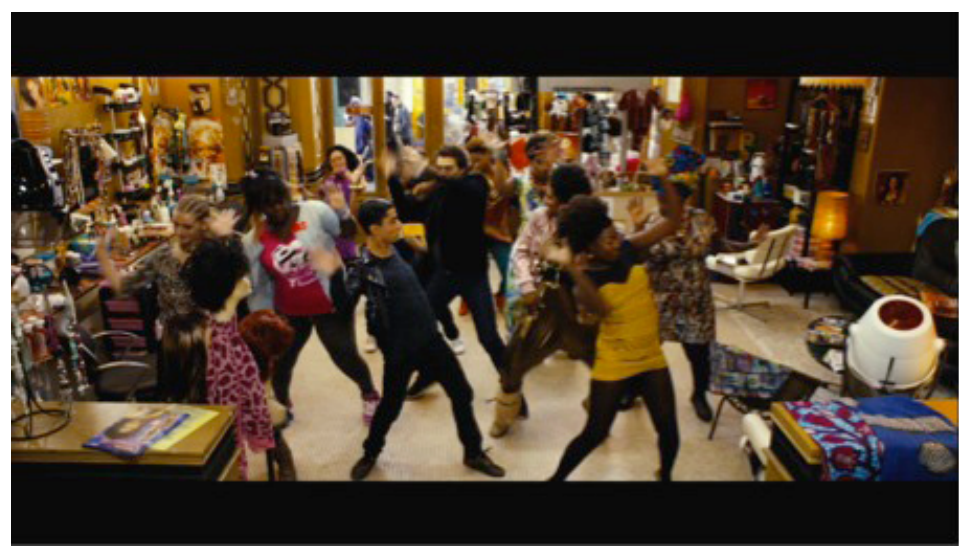

Figure 7. Plusieurs acteurs et actrices interprétant et dansant sur « Tout le monde » (21:12)

La deuxième chanson dont la mise en scène fait explicitement et uniquement référence aux sans-papiers est celle du groupe Téléphone, "Un autre monde », sortie en 1984. Là encore, un regard sur le paratexte permet de comprendre le choix d'Estrougo de ‘ réinvestir > (cf. Andrieu 2011) cette chanson dans son film : comme le rappelle Stéphane Hirschi dans son dernier livre sur La chanson française depuis 1980 (2016, 201), "Un autre monde " est « devenue suffisamment fédératrice pour venir conclure certains meetings politiques de Lionel Jospin en 2000 ", après que Jean-Louis Aubert avait chanté en juin 1981 place de la République pour célébrer la victoire de la gauche aux élections.

Dans Toi, moi, les autres, "Un autre monde » est interprétée par le personnage de Tina après qu'elle a été arrêtée par la police puis mise en prison, attendant d'être expulsée de France. Cette chanson, " chanson-personnage " selon la terminologie de Jérôme Rossi (2010, 66-67), répond elle aussi au patron de la comédie musicale classique : située à un moment stratégique de l'action, elle offre un commentaire du personnage-interprète sur sa situation (cf. Chion 2002, 6). L'esthétique froide de la mise en scène et les gros plans et très gros plans sur les visages des femmes (une seconde détenue interprète elle aussi la chanson) font d'autant mieux ressortir la brutalité de la situation, effet renforcé par la musique sur une guitare seule, et qui atteint son paroxysme dans la chute de la chanson, dont le texte s'énonce après un arrêt de la musique. Le < je > des canteuses devient un porte-voix pour les pensées de la plupart des sans-papiers, leurs motivations mais aussi et surtout leurs désillusions, l'imparfait pouvant se lire comme le signe que ce rêve est brisé - rêve brisé auquel fait écho la voix brisée de Tina. L'utopie du début s'est muée en une réalité terrible, Tina semble s'être résignée à ne plus voir que " les ombres » de ce monde dont elle /qu'elle avait rêvé.

La chanson et sa mise en scène jouent ici pleinement de ce que Michel Chion a appelé "l'effet de valeur ajouté » que la musique de film peut revêtir : 
la valeur ajoutée est cet effet en vertu duquel un apport d'information, d'émotion, d'atmosphère, amené par un élément sonore, est spontanément projeté par le spectateur (l'audio-spectateur, en fait) sur ce qu'il voit, comme si cela en émanait naturellement. (Chion 1995, 205)

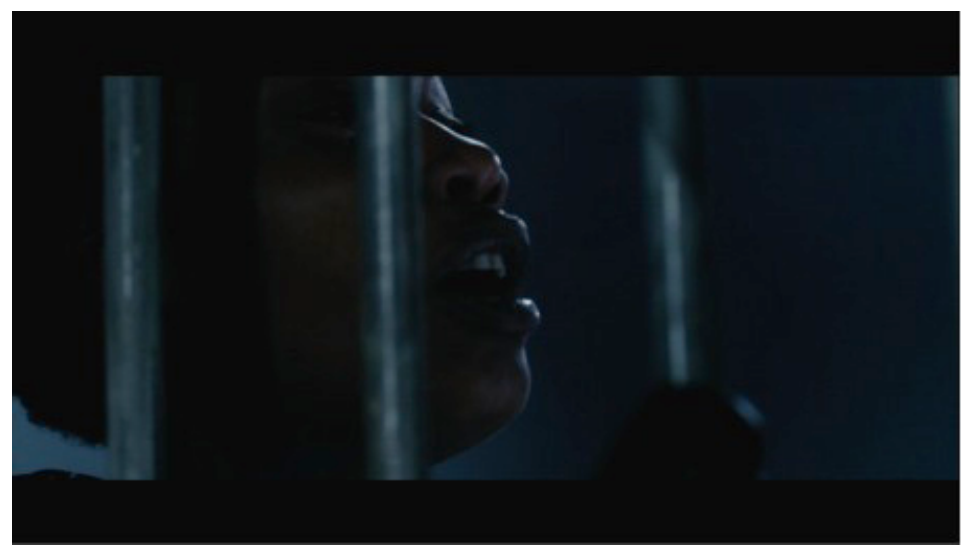

Figure 8. Tina interprétant « Un autre monde » (59:53)

La scène d' "Un autre monde " sous cette forme adaptée - et très émouvante - est à mon sens le seul moment de Toi, moi, les autres où la question des sans-papiers, sa narration, occupe le cœur de l'intrigue. Le recours à cette chanson tel qu'il est fait dans le film n'est alors pas sans rappeler un des emplois de la chanson au cinéma analysé par Joël July dans son article "De la chanson au cinéma ». Dans une partie intitulée "La chanson diégétisée ou chanson du cinéaste ", le stylisticien spécialiste de la chanson française souligne ainsi que :

La chanson propose donc en bonus une sorte de "supertexte " qui devient l'élément catalyseur du film, reflet synthétique de la morale que peut représenter pour le metteur en scène la fable cinématographique. Elle est certes l'illustration expressive d'un moment ponctuel de l'intrigue mais ses vertus (support musical, présence d'un refrain, émotion de la voix qui chante, structure close qui fabrique un microcosme, puissance réminiscente) lui permettent d'agir comme la forme épurée du destin des personnages, le révélateur du climat général ou la stylisation du message de l'auteur : la chanson est à prendre/entendre comme une métaphore. (July 2015, 246)

Là encore, le choix de cette chanson et son interprétation dans le récit de Toi, moi, les autres révèlent indéniablement une volonté critique. Toutefois, la suite du film dévoile que si, à ce moment précis, c'est bien le destin des personnages des sans-papiers qui est au cœur de l'intrigue, ce destin passera peu après à l'arrière-plan, pour être finalement effacé par celui des deux jeunes amoureux. Penchons-nous donc pour finir sur ce développement, auquel on ne peut nier une certaine perniciosité, perniciosité qui passe notamment par le recours aux chansons. 
Alors que la situation de Tina et Sally semble sans issue, le petit frère de Leïla, Ajib (Emir Seghir) commence de chanter "Sauver l'amour " de Daniel Balavoine (1985). Il se trouve devant l'église Saint-Bernard, lieu symbolique sur le fronton duquel est accrochée une banderole. Daniel Balavoine est lui aussi connu pour avoir été un chanteur engagé, Stéphane Hirschi rappelle qu'il « incarne vite, avec ses collègues Coluche et Michel Berger, la nouvelle figure de l'artiste engagé pour des causes humanitaires " (Hirschi 2016, 142), et notamment pour les pays d'Afrique subsaharienne. "Sauver l'amour " est d'ailleurs sorti la même année que le CD du projet "SOS Éthiopie ", en 1985. Il est frappant de remarquer que dans le film, si la voix principale est celle d'Ajib, la réalisatrice a décidé de garder les chœurs déjà présents dans la version originale de la chanson à partir du premier refrain. Là encore, les sentiments exprimés par le canteur à la troisième personne sont ceux ressentis par l'ensemble des immigrés concernés, ceux du film en tout cas, comme le suggère le montage alterné qui nous montre les sans-papiers dans le centre de rétention avant leur reconduite à la frontière. Il est donc évident que "l'espoir de voir enfin un jour meilleur ", " la peur immense de voir mourir ce sentiment d'amour intense ", le désir " d'exorciser la douleur ", comme le dit la chanson de Balavoine, sont des mots que la réalisatrice veut faire entendre à travers l'interprétation qu'en fait le jeune personnage. Mais une fois de plus, la narration du film nous rappelle que plus que des sans-papiers, c'est de Gabriel et Leïla, de leur histoire d'amour qu'il s'agit. Cette chanson est en effet interprétée à un moment du film où leur amour est mis en danger en raison de l'expulsion de Tina et Sally que Gabriel - comme le croit Leïla - n'a pu empêcher ; en outre, tous ses amis en veulent à Leïla, lui reprochant d'avoir abandonné Tina et Sally. Enfin, la chanson demande " Qu'est-ce qui pourrait sauver l'amour ? " et "Où est le sauveur ? „... faisant par-là même apparaitre Gabriel comme ce sauveur, puisque pendant la chanson, on le voit courir chercher Leïla pour lui annoncer, ainsi qu'à tous ses amis, qu'il sait comment empêcher l'expulsion de Tina et Sally.

Mais c'est sans doute dans la séquence finale du film que la difficulté à accorder à la question de la migration une importance telle qu'elle ne soit pas seulement un thème secondaire, devient complètement évidente. Cette séquence est composée de deux scènes. Dans la première, nous voyons comment Leilla, Gabriel et leurs amis parviennent à empêcher la reconduite au Sénégal de Tina et Sally en empêchant l'avion de décoller. Cette scène est presque entièrement chantée, les personnages interprètent "Quand on n'a que l'amour » de Jacques Brel. Là encore, la mise en scène joue à plein de l'effet empathique que peut avoir une musique et/ou une chanson dans un film, effet que Michel Chion définit comme «l'effet par lequel la musique adhère, ou semble directement adhérer, au sentiment dégagé par la scène, en particulier au sentiment supposé être ressenti par certains personnages " (Chion 1995, 228). Le titre même de la chanson, sa mise en scène ainsi que son interprétation signifient à nouveau à quel point le sujet des sans-papiers est rattaché à l'amour des deux protagonistes. D'un côté, la chanson établit littéralement un lien entre les deux niveaux de l'histoire : alors que les deux premières strophes chantent "mon amour toi et moi », cet amour s'ouvre petit à petit aux autres, «à ceux-là dont l'unique combat est de chercher le jour ». Les paroles de la chanson telle que celle-ci est mise en scène expriment bien que c'est l'amour de 
Leïla et Gabriel qui leur permet de "parler aux canons » et que, dans une sorte de mise en abîme, ils ont "une chanson pour convaincre un tambour ". D'un autre côté, cette idée de la seule force de l'amour est sans cesse soulignée par la caméra : la scène s'ouvre sur un plan rapproché des deux protagonistes assis l'un à côté de l'autre, puis filme leurs mains qui se rejoignent avant de les filmer en plan américain. Après cela, Leïla et Gabriel sont toujours filmés ensemble, alors que les autres personnages non. La scène évoque ainsi " ce moment fondamental ", selon Rick Altman, dans la comédie conte de fées : « celui où l'anecdote à caractère financier ou gouvernemental se dissout en anecdote amoureuse " (Altman 1992, 174). Les applaudissements des passagers de l'avion peuvent ainsi se lire aussi bien comme des applaudissements du < sauvetage > de Tina et Sally que du succès de l'histoire d'amour, répondant par là même à la question de savoir : "Qu'est-ce qui pourrait sauver l'amour ?"

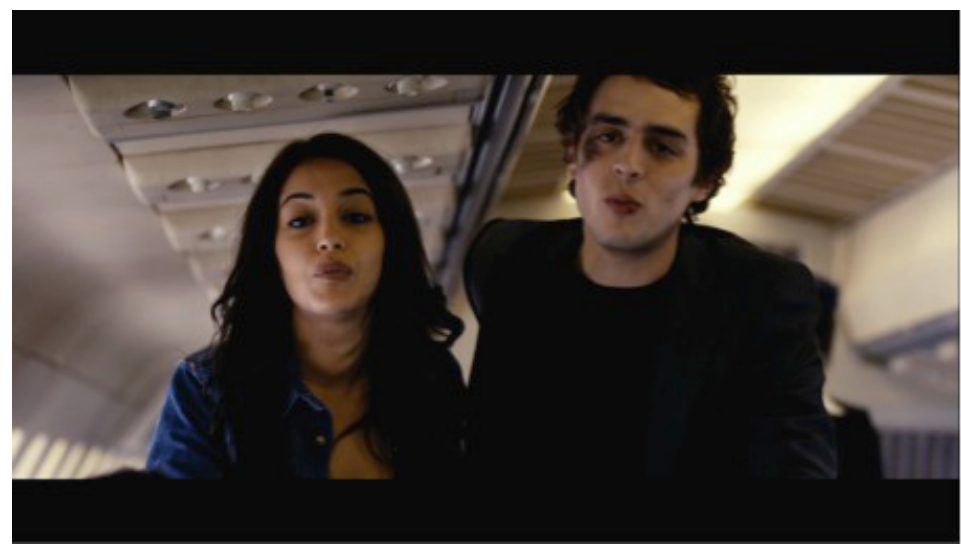

Figure 9. Leïla et Gabriel interprétant «Quand on n’a que l'amour » (1:17:12)

La dernière chanson du film, "Avec mes armes ", ne contredit pas ces observations. Écrite pour le film, c'est la chanson du générique de fin. Cas classique de la " chanson de film métadiscursive placée aux marges temporelles du récit ", cette chanson fonctionne comme " une parole narratoriale qui prolonge le contenu discursif issu des seuls dialogues " (July 2015, 243), dialogues mais aussi monologues et polylogues, qu'ils soient chantés ou non dans notre cas. "Avec mes armes " place elle aussi l'amour à l'origine de l'engagement social :

On cherche le bonheur avec des miettes de pain

On attend en vain que l'on nous prenne la main

La vie nous aveugle quand l'amour fait faux bond

Mais j'ai trouvé cause à effet papillon

J'm'engage pour l'amour plus que de raison

Puisque sur terre rien ne tourne rond 
Même si le soleil ne brille que pour toi

Ce ciel bleu blanc rouge on y a tous droit

Je me donne corps et âme avec mes armes

C'est ici et maintenant depuis l' temps que j'attends

Papapapa lalala

On se croit souvent de petite envergure

Faire partie d'une armée de soldats miniatures

Personne n'entend quand les soucis perdurent

Pourquoi pour certains le malheur a la dent dure

J'm'engage sans détour pour toutes ces raisons

Puisque sur terre rien ne tourne rond

Même si le soleil ne brille que pour toi

Ce ciel bleu blanc rouge on y a tous droit

Je me donne corps et âme avec mes armes

C'est ici et maintenant depuis l' temps que j'attends

Papapapa lalala

Selon la définition simple et concise des chansons engagées que propose Louis-Jean Calvet elles « illustrent les positions politiques d'un auteur, voire constituent des interventions dans le champ politique " (Calvet 2013, 192) ${ }^{4}$-, il est tout à fait possible de considérer "Avec mes armes " comme une chanson politique ou engagée. La mélodie joyeuse, les ‘ lallations > (les ‘ papallations `, pourrait-on dire) ainsi que le fait que la chanson soit reléguée au générique de fin permettent toutefois de faire douter de l'effet qu'elle aura sur les consciences des spectateurs et des spectatrices.

\section{Conclusion}

Conformément aux codes de la comédie conte de fées, le couple est réuni à la fin du film. Celui-ci nous laisse également comprendre que le conflit qui avait menacé l'amour des deux protagonistes est lui aussi résolu. Il s'agit on l'a vu du schéma classique des comédies musicales contes de fées, où la réussite d'un projet commun est étroitement liée à la réussite de l'histoire d'amour. Mais c'est justement cela qui apparaît particulièrement pernicieux pour un film qui se veut aborder une question aussi sérieuse et difficile que celle des sans-papiers : en effet, le film ne dit pas du tout ce qu'il advient de Tina et Sally. On les voit pour la dernière fois dans cette scène à la descente de l'avion - et on voit Tina agenouillée devant Leîla et Gabriel, leur prenant les mains, comme si elle se prosternait devant le Sauveur (!). 


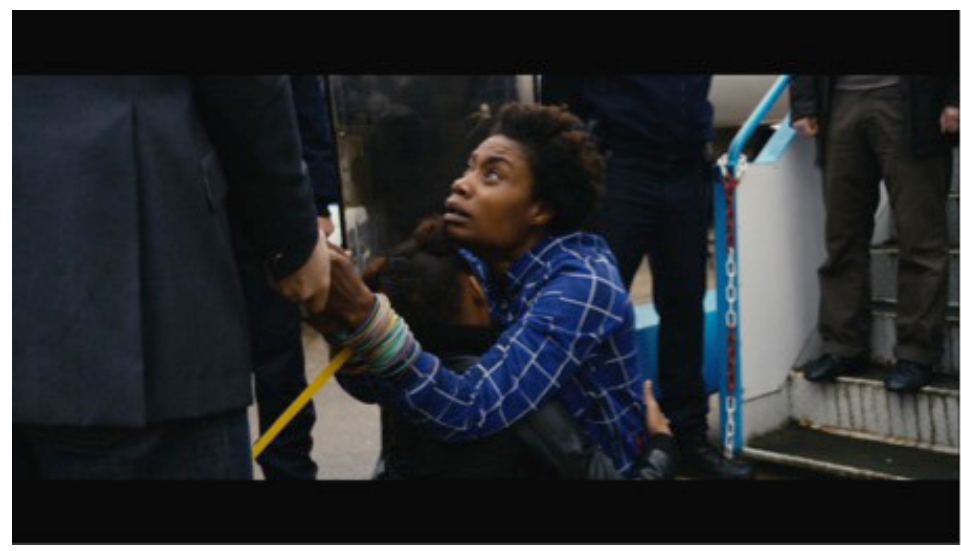

Figure 10. Tina à la descente de l'avion (1:19:13)

Il y a donc bien un happy end, mais il est réservé à Leïla et Gabriel. Plus encore, l'histoire de Tina et Sally apparaît finalement comme un faire-valoir de l'histoire d'amour entre Leilla et Gabriel. Le film s'était ouvert sur Leiila et Gabriel, à l'époque encore séparés par un montage alterné et des écrans partagés, il se termine sur une scène où les deux protagonistes sont réunis et dans laquelle Gabriel explique à Leïla que c'est grâce à lui que l'expulsion de Tina et Sally a pu être évitée. Outre la dimension problématique dans une perspective genrée ${ }^{5}-$ c'est l'homme qui réussit à convaincre la femme de se réinscrire au concours d'avocate, et c'est grâce à l'homme que le drame a pu être évité -, cette dernière scène montre à quel point l'histoire de l'injustice des sans-papiers n'est finalement que greffée à l'histoire d'amour, empêchant ainsi le discours sur la migration de se déployer pleinement. ${ }^{6}$ Nous constatons donc qu'en exploitant le patron romantique fictionnel sur fond de crise sociopolitique réelle, Toi, moi, les autres reprend une autre caractéristique fondamentale du film de genre, notamment de comédie musicale hollywoodienne, à savoir son rôle " fonctionnel ». Comme le souligne Rick Altman, "le film de genre pose des questions et résout des problèmes (ou du moins semble le faire) que la société a mis de côté parce qu'elle refuse de les régler ou ne sait comment s'y prendre "(Altman 1992, 357, c'est moi qui souligne).

Toi, moi, les autres se présente finalement comme un de ces « films juke-box, saupoudrés de hits hétérogènes" (Andrieu 2011, 127). La réalisatrice a donc exploité la dimension mémorielle - et par-là même émotionnelle - que peuvent revêtir les chansons préexistantes réinvesties dans un film au profit d'un discours romantique mais au détriment d'un discours politique. Son film n'aurait pu trouver de qualificatif plus juste que celui de ‘ conte social `.

\section{Notes}

1 Après Regarde-moi (2007), dans lequel joue d'ailleurs Marie-Sohna Condé.

2 Le fait que Benjamin Siksou, l'acteur interprétant Gabriel, ait été finaliste de l'émission télécrochet « Nouvelle Star» (en 2008) va dans ce sens dans la mesure où ce genre d'émission joue 
lui aussi pleinement avec la dimension patrimoniale et mémorielle du répertoire chansonnier français.

3 Céline Fontana rappelle d'ailleurs que l'on reproche à Zazie ses « combats consensuels, ses attaques trop lisses " (Fontana 2007, 204).

4 Je renvoie ici au chapitre « Chansons politiques, chansons engagées » dans son ensemble (Calvet 2013, 191-235).

5 Pour une analyse de la perniciosité de Toi, moi, les autres concernant les représentations genrées, je renvoie à Lagabrielle 2014.

6 Ma lecture du film sur ce point differe donc de celle proposée par David Pettersen, qui propose une interprétation inverse : "It is revealing that the end of the film abruptly shifts to a documentary mode, interweaving televised coverage of protests against deportation with the film's narrative resolution in which Gabriel and Leilla prevent the deportation of a friend. The jarring juxtaposition of modes reveals how Estrougo refuses to allow the social issues of the banlieue to disappear behind the genre conventions of the musical. » (Pettersen 2014, 48)

\section{Bibliographie}

Altman, Rick : La comédie musicale hollywoodienne. Les problèmes de genre au cinéma. Paris : Armand Colin 1992.

Andrieu, Michaël : Réinvestir la musique. Autour de la reprise musicale et de ses effets au cinéma. Paris : L'Harmattan, 2011.

Calvet, Louis-Jean : Chansons. La bande-son de notre histoire. Paris : L'Archipel, 2013.

Chion, Michel : La comédie musicale. Paris : Les Cahiers du cinéma, 2002.

Chion, Michel : La musique au cinéma. Paris : Fayard, 1995.

Dossier de presse : Toi, moi, les autres (2011). In : https:/www.unifrance.org/film/31470/toi-moiles-autres (consultation 05.01.2018).

Fontana, Céline : La chanson française. Paris : Hachette, 2007.

Harrod, Mary : From France with Love. Gender and Identity in French Romantic Comedy. London/ New York : I.B. Tauris, 2015.

Hirschi, Stéphane : Chanson. L’art de fixer le temps. De Béranger à Mano Solo. Valenciennes : Les belles lettres/Presses universitaires de Valenciennes, 2008.

Hirschi, Stéphane : La chanson française depuis 1980. De Goldman à Stromae, entre vinyle et mp3. Valenciennes : Les belles lettres/Presses universitaires de Valenciennes, 2016.

Johannès, Frank : «Brice Hortefeux condamné pour injure raciale ». In : Le Monde (04.06.2010), www.lemonde.fr (consultation 05.01.2018).

July, Joël : Esthétique de la chanson française contemporaine. Paris : L'Harmattan, 2007.

July, Joël : « De la chanson au cinéma ». In : Argod-Dutard, Françoise (éd.) : Le français en chantant. Septièmes rencontres de Liré. Rennes : Presses Universitaires de Rennes, 2015, 239-253.

Lagabrielle, Renaud : "Connaît-on la chanson ? Le film en chanté, un genre en devenir du cinéma français ». In : Contemporary French Civilization 41,3-4 (2016), 489-501. 
Lagabrielle, Renaud : "Boys Meet Girls, Paris Meets Hollywood. Quelques rendez-vous manqués de films français en chanté ». In : Diogène 245 (janvier-mars 2014), 177-195.

Pettersen, David : «American Genre Film in the French Banlieue : Luc Besson and Parkour ». In : Cinema Journal 53,3 (Spring 2014), 26-51.

Powrie, Phil : «Les chansons préexistantes dans le cinéma français actuel ». In : Abhervé, Sandrine /

Binh, N.T. / Moure, José (éds) : Musiques de films. Nouveaux enjeux. Rencontre sensible entre deux arts. Paris : Les Impressions Nouvelles, 2014, 46-59.

Rossi, Jérôme : "La chanson au cinéma : proposition d'une triple typologie ». In : Pistone, Danièle (éd.) : Corpus et typologies. Paris : Université de Paris Sorbonne, 2010, 59-81.

Wagner, Birgit : «Mirages du récit. La voix plurielle d'Alger dans Les oranges d'Aziz Choukri ». In : Bouchentouf-Siagh, Zohra (éd.) : Dzayer, Alger. Ville portée, rêvée, imaginée. Alger : Casbah Editions, 2006, 135-146.

\section{Filmographie}

Estrougo, Audrey (dir.) : Toi, moi, les autres. France, 2011 (DVD). 